\title{
Hard-Sphere Fluids in Contact with Curved Substrates
}

\author{
P. Bryk, ${ }^{1,2,3}$ R. Roth, ${ }^{2,3}$, K.R. Mecke, ${ }^{2,3}$, and S. Dietrich, $, 2,3$ \\ 1 Department for the Modeling of Physico-Chemical Processes, Maria Curie-Sktodowska University, 20-031 Lublin, Poland \\ 2 Max-Planck-Institut für Metallforschung, Heisenbergstrasse 3, D-70569 Stuttgart, Germany \\ ${ }^{3}$ Institut für Theoretische und Angewandte Physik, Universität Stuttgart, Pfaffenwaldring 57, D-70569 Stuttgart, Germany
}

\begin{abstract}
The properties of a hard-sphere fluid in contact with hard spherical and cylindrical walls are studied. Rosenfeld's density functional theory (DFT) is applied to determine the density profile and surface tension $\gamma$ for wide ranges of radii of the curved walls and densities of the hard-sphere fluid. Particular attention is paid to investigate the curvature dependence and the possible existence of a contribution to $\gamma$ that is proportional to the logarithm of the radius of curvature. Moreover, by treating the curved wall as a second component at infinite dilution we provide an analytical expression for the surface tension of a hard-sphere fluid close to arbitrary hard convex walls. The agreement between the analytical expression and DFT is good. Our results show no signs for the existence of a logarithmic term in the curvature dependence of $\gamma$.
\end{abstract}

\section{INTRODUCTION}

The properties of inhomogeneous fluids with spherical or cylindrical geometries have been investigated by many authors in recent years [1-9] because curvature plays an important role in many physical situations, including the following examples. In the context of nucleation phenomena [10-12] one considers the spontaneous formation of spherical droplets in a super-saturated vapor. The variation of the local curvature of confinements leads to a more complicated form of the depletion forces in colloidal suspensions [13-15]. Wetting or critical adsorption of a fluids on curved substrates, such as spherical or cylindrical colloidal particles may lead to the formation of liquid bridges if two colloids come close to each other, and subsequently to flocculation $[4,7,16-19]$. In studies of the hydrophobic effect one is concerned with the cost in free energy to immerse a hydrophobic particle into a liquid [20,21]. Despite these efforts, the properties of curved fluid interfaces are not understood as fully as the planar one. Whether one deals with a drop of liquid surrounded by its vapor or with a big spherical particle immersed into a solvent of small particles, the statistical-mechanical description of such systems generates additional difficulties [22,23] which call for further studies [24-26].

Within this context, here we consider the case of a hard-sphere fluid in the grand canonical ensemble, characterized by the radius $R$ of the spheres and the bulk number density $\rho$ or equivalently the bulk packing fraction $\eta=(4 \pi / 3) R^{3} \rho$, in contact with a hard spherical or cylindrical wall. These curved walls can either be viewed as an external potential exerted on the hard-sphere fluid,

$$
V_{i}^{e x t}(r)= \begin{cases}0, & r>R_{i} \\ \infty, & r<R_{i}\end{cases}
$$

where the subscript $i=s, c$ denotes the spherical and cylindrical symmetry, respectively, or as the surfaces of a second (big) species of radius $R_{i}^{(b)}$. The radii $R_{i}$ entering the external potential in Eq. (1) are related to the actual radii $R_{i}^{(b)}$ via $R_{i}=R_{i}^{(b)}+R$ (see Fig. 1 ).

The grand canonical potential of the system $\Omega_{i}$ can be divided into a bulk part $\Omega_{i}^{b u l k}=-p V_{i}$, where $p$ is the pressure of the hard-sphere fluid and $V_{i}$ is the volume accessible to the system, and an excess (surface) part $\Omega_{i}^{e x}$ : $\Omega_{i}=\Omega_{i}^{b u l k}+\Omega_{i}^{e x}$. The surface tension $\gamma_{i}$ is readily defined as the excess grand potential per unit area,

$$
\gamma_{i}=\frac{\Omega_{i}^{e x}}{A_{i}^{(b)}}=\frac{\Omega_{i}-\Omega_{i}^{b u l k}}{A_{i}^{(b)}}
$$

where $A_{i}^{(b)}$ is the surface area of the curved wall. While $\Omega_{i}$ is uniquely defined this division into bulk and surface excess parts is not unique. Depending on the choice of the dividing surface the surface tension can even change sign. As indicated by the superscript (b) in Eq. (2) we use the actual wall corresponding to the radius $R_{i}^{(b)}$ as the dividing interface.

Within density functional theory (DFT), the grand potential $\Omega_{i}$ of the system can be expressed as

$$
\Omega_{i}[\rho]=\mathcal{F}[\rho]+\int d^{3} r \rho(\mathbf{r})\left(V_{i}^{e x t}(\mathbf{r})-\mu\right),
$$


where $\mathcal{F}[\rho]=\mathcal{F}_{\text {ex }}[\rho]+\mathcal{F}_{i d}[\rho]$ is the intrinsic free energy functional that can be split into an ideal gas contribution $\mathcal{F}_{i d}[\rho]$ and an excess (over the ideal gas) term $\mathcal{F}_{\text {ex }}[\rho]$. The only explicit dependence of $\Omega_{i}[\rho]$ on the external potential originates from the second term in Eq. (3). The equilibrium density profile $\rho(\mathbf{r})$ satisfies the Euler-Lagrange equation

$$
\frac{\delta \Omega_{i}[\rho]}{\delta \rho(\mathbf{r})}=0 .
$$

For the equilibrium density profile $\rho(\mathbf{r})$ the density functional reduces to the grand potential of the system, i.e., $\Omega_{i}=\Omega_{i}[\rho(\mathbf{r})]$, and we can rewrite Eq. $(2)$ as

$$
\gamma_{i}=\frac{\Omega_{i}[\rho(\mathbf{r})]+p V_{i}}{A_{i}^{(b)}} .
$$

From Eqs. (1), (3), and (4) it follows that the change in the grand potential due to an infinitesimal change of the radius $R_{i}^{(b)}$ of the curved wall, at constant chemical potential $\mu$ and temperature $T$, is given by

$$
\left(\frac{\partial \Omega_{i}}{\partial R_{i}^{(b)}}\right)_{\mu, T}=\int d^{3} r \frac{\delta \Omega_{i}[\rho]}{\delta \rho(\mathbf{r})} \frac{\partial \rho(\mathbf{r})}{\partial R_{i}^{(b)}}+\int d^{3} r \rho(\mathbf{r}) \frac{\partial V_{i}^{e x t}(\mathbf{r})}{\partial R_{i}^{(b)}} .
$$

The first term on the right-hand side of Eq. (6) vanishes by virtue of Eq. (4) while the second term, for the external potential given in Eq. (1), is equal to $\beta^{-1} A_{i} \rho\left(R_{i}^{+}\right)$, where $\rho\left(R_{i}^{+}\right)$denotes the contact density of the hard-sphere fluid at the curved wall. This leads to the sum rules [24]

$$
\beta\left(\frac{\partial \Omega_{i}}{\partial R_{i}^{(b)}}\right)_{\mu, T}= \begin{cases}4 \pi R_{s}^{2} \rho\left(R_{s}^{+}\right), & \text {for a spherical wall } \\ 2 \pi R_{c} L \rho\left(R_{c}^{+}\right), & \text {for a cylindrical wall. }\end{cases}
$$

In the case of a cylindrical wall we consider the thermodynamic limit $\lim _{L \rightarrow \infty}\left(\Omega_{c} / L\right)$ so that effects due to a finite length $L$ of the cylinder drop out. We note that the sum rules in Eq. (7) are valid for all one-component fluids in contact with hard spherical or cylindrical walls and are satisfied by all density functionals within weighted-density approximation (WDA) [2].

Using the actual radius $R_{i}^{(b)}$ also as the radius of the dividing interface (see Fig. 1), the grand potential $\Omega_{i}=$ $-p V_{i}+\gamma_{i} A_{i}^{(b)}$ of the system is separated into a bulk and surface term with the accessible volume

$$
V_{i}=V_{i}(\bar{R})-V_{i}\left(R_{i}^{(b)}\right),
$$

where $V_{s}(r)=\frac{4 \pi}{3} r^{3}, V_{c}(r)=\pi r^{2} L$, and $\bar{R}$ is a macroscopicly large radius considered in the thermodynamical limit, and the surface areas

$$
A_{i}^{(b)}= \begin{cases}4 \pi\left(R_{s}^{(b)}\right)^{2}, & \text { for a spherical wall } \\ 2 \pi R_{c}^{(b)} L, & \text { for a cylindrical wall } .\end{cases}
$$

The sum rule in Eq. (7) can then be expressed as

$$
\rho\left(R_{s}^{+}\right)\left(\frac{R_{s}}{R_{s}^{(b)}}\right)^{2}=\beta p+\frac{2 \beta \gamma_{s}\left(R_{s}^{(b)}\right)}{R_{s}^{(b)}}+\beta\left(\frac{\partial \gamma_{s}\left(R_{s}^{(b)}\right)}{\partial R_{s}^{(b)}}\right)_{\mu, T}
$$

for a spherical wall and

$$
\rho\left(R_{c}^{+}\right) \frac{R_{c}}{R_{c}^{(b)}}=\beta p+\frac{\beta \gamma_{c}\left(R_{c}^{(b)}\right)}{R_{c}^{(b)}}+\beta\left(\frac{\partial \gamma_{c}\left(R_{c}^{(b)}\right)}{\partial R_{c}^{(b)}}\right)_{\mu, T}
$$

for a cylindrical wall. Equations (10) and (11) indicate that different versions of DFTs might lead to different contact values near curved walls even if the underlying equation of state is the same, which leads to identical contact values at a hard planar wall. As pointed out by J.R. Henderson [26], these sum rules have interesting implications. If $\rho\left(R_{i}^{+}\right)$ is analytic as a function of $R_{i}^{-1}=\left(R_{i}^{(b)}+R\right)^{-1}$ one has 


$$
\rho\left(R_{i}^{+}\right)=c_{0}+\frac{c_{i}^{(1)}}{R_{i}^{(b)}+R}+\frac{c_{i}^{(2)}}{\left(R_{i}^{(b)}+R\right)^{2}}+\frac{c_{i}^{(3)}}{\left(R_{i}^{(b)}+R\right)^{3}}+\cdots
$$

with $c_{0}=\beta p$ for both the spherical and the cylindrical wall and expansion coefficients $c_{i}^{(j)}$ for $j=1,2, \ldots$. Note that the expansion in Eq. (12) is assumed to converge for arbitrary non-zero values of $R_{i}$. This assumption will be corroborated by our numerical results in Sec. III. With Eq. (12) the solution of the differential equation (10) can be written as

$$
\beta \gamma_{s}\left(R_{s}^{(b)}\right)=\frac{C_{s}^{(1)}}{2}\left[1+\frac{2 C_{s}^{(2)}}{C_{s}^{(1)}} \frac{1}{R_{s}^{(b)}}+\frac{2 c_{s}^{(3)}}{C_{s}^{(1)}} \frac{\ln \left(R_{s}^{(b)} / R\right)}{\left(R_{s}^{(b)}\right)^{2}}+\frac{2 D_{s}}{C_{s}^{(1)}} \frac{1}{\left(R_{s}^{(b)}\right)^{2}}+\mathcal{O}\left(\frac{1}{\left(R_{s}^{(b)}\right)^{3}}\right)\right]
$$

where $D_{s}$ is an integration constant, $C_{s}^{(1)}=c_{s}^{(1)}+2 \beta p R$ and $C_{s}^{(2)}=c_{s}^{(2)}+c_{s}^{(1)} R+\beta p R^{2}$. Likewise, with Eq. (12) the solution of the differential equation (11) can be written as

$$
\beta \gamma_{c}\left(R_{c}^{(b)}\right)=C_{c}^{(1)}\left[1+\frac{c_{c}^{(2)}}{C_{c}^{(1)}} \frac{\ln \left(R_{c}^{(b)} / R\right)}{R_{c}^{(b)}}+\frac{D_{c}}{C_{c}^{(1)}} \frac{1}{R_{c}^{(b)}}+\mathcal{O}\left(\frac{1}{\left(R_{c}^{(b)}\right)^{2}}\right)\right],
$$

where $D_{c}$ is an integration constant and $C_{c}^{(1)}=c_{c}^{(1)}+\beta p R$. In the case of the spherical wall $\gamma_{s}\left(R_{s}^{(b)}\right)$ has the form

$$
\beta \gamma_{s}\left(R_{s}^{(b)}\right)=\beta \gamma_{s}(\infty)\left[1-\frac{2 \delta_{T}}{R_{s}^{(b)}}+\mathcal{O}\left(\frac{\ln \left(R_{s}^{(b)} / R\right)}{\left(R_{s}^{(b)}\right)^{2}}\right)\right]
$$

where $\gamma_{s}(\infty)=\beta^{-1} \frac{c_{s}^{(1)}}{2}+p R$ is the surface tension of the planar wall and

$$
\delta_{T}=-\frac{c_{s}^{(2)}+c_{s}^{(1)} R+\beta p R^{2}}{c_{s}^{(1)}+2 \beta p R}
$$

is the so-called Tolman length [27], which plays an important role in nucleation theory [4,10-12]. We note that the value of the Tolman length depends on the choice of the dividing surface; here the dividing surface has the radius $R_{s}^{(b)}$. If these logarithmic terms in the expansion of the surface tensions do not vanish, the concept of the Tolman length apparently breaks down for cylindrically curved and generally shaped walls $[25,26]$.

The analyticity of $\gamma(R)$ in terms of $\frac{1}{R}$ is of considerable importance because it provides the basis for the so-called Helfrich expansion of the surface free energy of arbitrarily curved surfaces in powers of the principal curvatures [28,29]. Although this approach appears to be very useful for describing membranes [30] it has been shown recently that the presence of long-ranged dispersion forces destroys the aforementioned analyticity for fluid interfaces $[4,31,32]$ and prevents the use of a Helfrich-type interface Hamiltonian for such systems. The possible occurrence of logarithmic terms in Eqs. (13) and (14) following from the simple expansion (12) raises the issue whether the absence of analyticity is not only due the presence of long-ranged forces but is an intrinsically geometrical effect which shows up even for surface free energies of hard-sphere fluids. The present study aims at clarifying this conceptual point.

To this end we investigate the curvature dependence of the surface tension as well as relationships between thermodynamic and local properties of the hard-sphere fluids following two different routes. First, in Sec. II the surface tension is determined from a bulk theory in which the curved wall is considered as the surface of an additional component at infinite dilution. Second, a numerical approach based on the minimization of the Rosenfeld fundamental measure theory (FMT) free energy functional [33,34] is presented in Sec. III. In this approach, the hard-sphere fluid is considered to be exposed to the external potential of Eq. (1).

\section{BULK THEORY}

We start by considering the bulk of a one component hard-sphere fluid in a volume $V_{\text {tot }}$ with $V_{\text {tot }} \rightarrow \mathbb{R}^{3}$ in the thermodynamic limit. The grand potential of this system is given by $\Omega_{0}=-p V_{t o t}$, where $p$ is the pressure of the hard-sphere fluid. A single particle of a second component $b$ is inserted into this system. In view of the following discussion the only restriction for this particle is that it must be hard and convex. The grand potential of the new system is

$$
\Omega=\Omega_{0}+\Delta \Omega,
$$


where $\Delta \Omega$ measures the change of the grand potential due to the insertion of a single particle. $\Delta \Omega$ equals the oneparticle direct correlation function $[14,15,35]$ which in the bulk limit considered here is simply the excess chemical potential of species $b$ in the dilute limit $\rho_{b} \rightarrow 0$, i.e.,

$$
\beta \Delta \Omega=-c_{b}^{(1)} .
$$

Although Eqs. (17) and (18) are formally exact it is important to notice that this approach within a bulk theory is expected to only work reliably if the considered fluid is in a single phase. In the case of phase separation, which could lead to wetting or drying of the big sphere, this approach most likely would fail [36].

We evaluate Eq. (18) within the framework of Rosenfeld's Fundamental Measure Theory (FMT). Within this approach $[33,34]$ the excess free energy functional (over the ideal gas) is given by

$$
\beta \mathcal{F}_{e x}=\int d^{3} r \Phi\left(\left\{n_{\alpha}\right\}\right)
$$

where $n_{\alpha}$ denote weighted densities

$$
n_{\alpha}(\mathbf{r})=\sum_{j=1}^{N} \int d^{3} r^{\prime} \rho_{j}\left(\mathbf{r}^{\prime}\right) w_{\alpha}^{(j)}\left(\mathbf{r}-\mathbf{r}^{\prime}\right)
$$

with geometrical weight functions $w_{\alpha}^{(j)}$ of species $j=1, \ldots, N$. In Rosenfeld's approach there are six different weighted densities, four are scalar and two are vector-like. For a general hard body $j$ one has $[37,38]$

$$
\begin{aligned}
w_{3}^{(j)}(\mathbf{r}) & =\Theta\left(\left|\mathbf{r}-\mathbf{R}^{(j)}\right|\right) \\
w_{2}^{(j)}(\mathbf{r}) & =\delta\left(\left|\mathbf{r}-\mathbf{R}^{(j)}\right|\right), \\
\mathbf{w}_{V 2}^{(j)}(\mathbf{r}) & =\hat{\mathbf{n}}^{(j)} \delta\left(\left|\mathbf{r}-\mathbf{R}^{(j)}\right|\right), \\
w_{1}^{(j)}(\mathbf{r}) & =\frac{H^{(j)}}{4 \pi} w_{2}^{(j)}(\mathbf{r}), \\
w_{0}^{(j)}(\mathbf{r}) & =\frac{K^{(j)}}{4 \pi} w_{2}^{(j)}(\mathbf{r}), \\
\mathbf{w}_{V 1}^{(j)}(\mathbf{r}) & =\frac{H^{(j)}}{4 \pi} \mathbf{w}_{V 2}^{(j)}(\mathbf{r}),
\end{aligned}
$$

where $\mathbf{R}^{(j)}=\mathbf{R}^{(j)}(\theta, \varphi)$ is the radius vector to the surface and $\hat{\mathbf{n}}^{(j)}$ is its outward unit vector normal to the surface at point $\mathbf{R}^{(j)}$ of particle $j . H^{(j)}$ and $K^{(j)}$ are the integrated mean curvature and integrated Gaussian curvature of the particle $j$, respectively $[37,38]$.

In the bulk limit for which the density profiles $\rho_{j}(\mathbf{r})$ are constant, as considered in this section, the vector weighted densities vanish, and Eq. (18) takes the form

$$
-c_{b}^{(1)}=\frac{\partial \Phi}{\partial n_{3}} \zeta_{3}+\frac{\partial \Phi}{\partial n_{2}} \zeta_{2}+\frac{\partial \Phi}{\partial n_{1}} \zeta_{1}+\frac{\partial \Phi}{\partial n_{0}} \zeta_{0},
$$

with characteristic functions $\zeta_{i}, i=0, \ldots, 3$ of the shape of the particle of species $b$.

Assuming the general functional of the given by Eqs. (19)-(21), one has for a general convex hard particle $[37,38]$

$$
\begin{aligned}
& \zeta_{3}=V^{(b)}, \\
& \zeta_{2}=A^{(b)}, \\
& \zeta_{1}=A^{(b)} \frac{H^{(b)}}{4 \pi},
\end{aligned}
$$

and

$$
\zeta_{0}=A^{(b)} \frac{K^{(b)}}{4 \pi},
$$

where the shape of the particle is specified by its volume $V^{(b)}$, its surface area $A^{(b)}$, and integrated curvatures $H^{(b)}$ and $K^{(b)}$. 
Within FMT there are several expressions for the excess free energy density $\Phi$. For the given problem we have chosen the original Rosenfeld form [33]

$$
\Phi\left(\left\{n_{\alpha}\right\}\right)=-n_{0} \ln \left(1-n_{3}\right)+\frac{n_{1} n_{2}-\mathbf{n}_{1} \cdot \mathbf{n}_{2}}{1-n_{3}}+\frac{n_{2}^{3}-3 n_{2} \mathbf{n}_{2} \cdot \mathbf{n}_{2}}{24 \pi\left(1-n_{3}\right)^{2}} .
$$

The partial derivatives of the excess free energy density with respect to the weighted densities in the dilute limit of species $b$ depend only on the packing fraction $\eta$ and the radius $R$ of the hard-sphere fluid:

$$
\begin{aligned}
\frac{\partial \Phi}{\partial n_{3}} & =\frac{3 \eta}{4 \pi R^{3}} \frac{1+\eta+\eta^{2}}{(1-\eta)^{3}} \equiv \beta p_{P Y}, \\
\frac{\partial \Phi}{\partial n_{2}} & =\frac{3 \eta(2+\eta)}{8 \pi R^{2}(1-\eta)^{2}} \equiv \beta \gamma_{H W}^{S P T}, \\
\frac{\partial \Phi}{\partial n_{1}} & =\frac{3 \eta}{R(1-\eta)} \equiv \phi_{1},
\end{aligned}
$$

and

$$
\frac{\partial \Phi}{\partial n_{0}}=-\ln (1-\eta) \equiv \phi_{0}
$$

In the above equations $p_{P Y}$ and $\gamma_{H W}^{S P T}$ are the Percus-Yevick compressibility equation of state and the scaled-particle theory (SPT) expression for the surface tension of a hard-sphere fluid at a planar hard wall [39], respectively.

With the characteristic functions in Eqs. (23)-(26) and the partial derivatives given by Eqs. (28)-(31) one finds within this approach for the grand potential of a hard-sphere fluid surrounding any convex hard particle $b$

$$
\beta \Omega=-\beta p_{P Y}\left(V_{t o t}-V^{(b)}\right)+A^{(b)}\left(\beta \gamma_{H W}^{S P T}+\frac{H^{(b)}}{4 \pi} \phi_{1}+\frac{K^{(b)}}{4 \pi} \phi_{0}\right) .
$$

By choosing the surface of the convex particle as the dividing interface, the bulk contribution to the grand potential is simply $\Omega_{0}=-p^{P Y}\left(V_{t o t}-V^{(b)}\right)$ and the surface tension follows directly as

$$
\beta \gamma\left(H^{(b)}, K^{(b)}\right)=\beta \gamma_{H W}^{S P T}+\frac{H^{(b)}}{4 \pi} \phi_{1}+\frac{K^{(b)}}{4 \pi} \phi_{0} .
$$

The particular choice of the dividing interface underlying Eq. (33) is motivated by the simplicity of the resulting expression for the surface tension. Other choices of the dividing interface would lead to significantly more complex expressions and the elegant, simple form of Eq. (33) would be lost. Obviously within this bulk approach it is impossible to pick up a contribution to the surface tension that is proportional to the logarithm of the radius of curvature.

The surface tension in Eq. (33) is always positive for a non-zero packing fraction $\eta$ and its change for small curvature due to an increase in curvature is described by the Tolman length $\delta_{T}$ which within this approach is defined by

$$
\gamma\left(H^{(b)}, K^{(b)}\right)=\gamma_{H W}^{S P T}\left(1-2 H^{(b)} \delta_{T}+\mathcal{O}\left(K^{(b)}\right)\right),
$$

so that it is independent of the shape of the convex particle with

$$
\delta_{T}=-\frac{\phi_{1}}{8 \pi \beta \gamma_{H W}^{S P T}}=\frac{\eta-1}{\eta+2} R .
$$

Thus within this approach we find that the generalized Tolman length for a general hard convex cavity is negative, provided the surface of this hard cavity is chosen as the dividing surface. The grand potential (Eq. (32)) and the surface tension (Eq. (33)) have been derived for a general convex hard wall and are related to the Helfrich Hamiltonian [28]. In order to be able to calculate the contact density of a hard-sphere fluid on the basis of the sum rule in Eq. (7) one has to evaluate the expression in Eq. (33) for special cases. For a big spherical particle the mean curvature $H_{s}^{(b)}=1 / R_{s}^{(b)}$ and the Gaussian curvature $K_{s}^{(b)}=1 /\left(R_{s}^{(b)}\right)^{2}$. By combining Eqs. (33) and (10) within the present bulk theory one finds for the contact value of the density profile of a hard-sphere fluid at a big hard sphere:

$$
\rho\left(R_{s}^{+}\right)=\beta p_{P Y}-\frac{9 \eta^{2}}{4 \pi(1-\eta)^{3} R^{3}}\left\{(1+\eta) \frac{R}{R_{s}}-\eta \frac{R^{2}}{R_{s}^{2}}\right\} .
$$


For finite curvatures the contact density is smaller than in the planar limit, which in the present approach is given by the Percus-Yevick compressibility pressure $\beta p_{P Y}$. The difference between the contact density at finite curvature and its planar wall limit is a quadratic function in $1 / R_{s}$.

For a cylindrical wall we have to proceed more carefully. Since Eq. (33) was derived assuming a finite convex body we take the limit $L \rightarrow \infty$ only after the cylinder is inserted into the bulk system. In this limit the mean curvature $H_{c}^{(b)}=1 /\left(2 R_{c}^{(b)}\right)$ and the Gaussian curvature $K_{c}^{(b)}=0$. The contact density of a hard-sphere fluid close to a cylindrical wall follows from Eq. (33) and the sum rule in Eq. (11) as

$$
\rho\left(R_{c}^{+}\right)=\beta p_{P Y}-\frac{9 \eta^{2}}{4 \pi(1-\eta)^{3} R^{3}}\left\{\frac{(1+\eta)}{2} \frac{R}{R_{c}}\right\}
$$

which is linear in $1 / R_{c}$. For large radii of curvature $R_{i} \rightarrow \infty$ the contact densities at a big sphere $\rho\left(R_{s}^{+}\right)$and at a cylinder $\rho\left(R_{c}^{+}\right)$are related by $2 \rho\left(R_{c}^{+}\right)-\rho\left(R_{s}^{+}\right)=\beta p_{P Y}+\mathcal{O}\left(\frac{1}{R_{s}^{2}}\right)$.

\section{NUMERICAL RESULTS}

As mentioned in the Introduction an alternative approach for calculating the surface tension and the contact density of a hard-sphere fluid at a spherical or cylindrical wall consists of minimizing the density functional, Eq. (3), numerically. Within this approach the curved wall enters the problem via the external potential specified in Eq. (1). The solution of the corresponding Euler-Lagrange equation yields the inhomogeneous equilibrium density profile $\rho(r)$. From $\rho(r)$ both the contact densities $\rho\left(R_{i}^{+}\right)=\rho\left(r=R_{i}^{+}\right)$and the surface tensions $\gamma_{i}\left(R_{i}^{(b)}\right)$, Eq. (5), follow directly. In order to verify that the numerical DFT results for the contact density and the surface tension (from minimization) are consistent and to probe the numerical accuracy of our DFT calculation, we confirmed numerically that the sum rule in Eq. (7) is satisfied very accurately (within the range of four to six significant digits depending on the packing fraction).

Although the minimization of the density functional and the bulk route discussed in Sec. II are in principal equivalent approaches, they differ in practice because the actual density functional for the hard-sphere fluid is an approximation. Within an approximate treatment it is expected that the numerical solution of the Euler-Lagrange equation, which is based on a variational principle, is more accurate than the aforementioned bulk approach. Nevertheless, for the hard-sphere fluid which, apart from freezing, exhibits only a single phase both routes are expected to be in qualitative or even semi-quantitative agreement with each other.

First we present in Fig. 2 our numerical results for the surface tension at a spherical wall as obtained by full minimization of the Rosenfeld functional for values of $\eta$ in the range between $\eta=0.1$ and $\eta=0.42$ and with radii of curvature $R_{s}^{(b)}$ in the range $R_{s}^{(b)}=R$ and $R_{s}^{(b)}=10000 R$. The DFT results are denoted by symbols and the predictions of the bulk theory by full lines. For small values of $\eta$ we find both results to be in excellent, quantitative agreement for all curvatures, see Fig. 2(a). Close to the planar wall limit the level of quantitative agreement reduces significantly for larger packing fractions, while it remains good for large curvatures. This deviation between the two different routes is expected because in the limit $R_{s}^{(b)} \rightarrow \infty$ the bulk route reduces to the SPT prediction of the surface tension at a planar wall, which is known to overestimate the surface tension of a hard-sphere fluid at a planar hard wall [40]. Since SPT obtains the planar wall limit from extrapolating the behavior at small cavity sizes [39] this deviation is no surprise. Recently it was suggested that SPT can be re-optimized by taking the planar wall limit into account more accurately [36].

Even for packing fractions at which the level of quantitative agreement is moderate (see Fig. 2(b)), there is a good qualitative agreement between the DFT results and our bulk approach. This implies that the curvature dependence of our numerical data of $\gamma_{s}$ can be best approximated by a polynomial quadratic in $1 / R_{s}^{(b)}$ with no evidence for a logarithmic term.

The DFT results for the surface tension of a hard-sphere fluid in contact with a hard cylindrical wall are shown in Fig. 3. Again the quantitative agreement between the DFT results (symbols) and predictions of the bulk theory (full lines) are excellent for low packing fractions and fair at higher values of $\eta$. The curvature dependence of the DFT data is captured very well by a function linear in $1 / R_{c}^{(b)}$ as predicted by the bulk route.

A remarkable feature of Eq. (33) is that the ratio of the coefficients corresponding to the term of the leading order in $1 / R_{i}$ for spherical and cylindrical symmetry is exactly 2 (since $H_{s}^{(b)}=2 H_{c}^{(b)}$ ). This behavior can also be found in the numerical results of DFT calculations, i.e., based on the inhomogeneous density profiles $\rho(r)$. In Table I we show values of least-square fits assuming that $\beta \gamma_{s}\left(R_{s}^{(b)}\right)=a_{s}^{(0)}+\frac{a_{s}^{(1)}}{R_{s}^{(b)}}+\frac{a_{s}^{(2)}}{\left(R_{s}^{(b)}\right)^{2}}$ and $\gamma_{c}\left(R_{c}^{(b)}\right)=a_{c}^{(0)}+\frac{a_{c}^{(1)}}{R_{c}^{(b)}}$, i.e., the simplest 
formulae to fit our DFT data. To a good approximation the ratio $a_{s}^{(1)} / a_{c}^{(1)}$ equals 2 for all densities. This ratio agrees with the predictions of the bulk theory; $a_{s}^{(0)}=a_{c}^{(0)}$ is the planar wall surface tension. The Tolman length (compare Eq. (34)) is given by $\delta_{T}^{(s)}=-a_{s}^{(1)} /\left(2 a_{s}^{(0)}\right)$ and $\delta_{T}^{(c)}=-a_{c}^{(1)} / a_{c}^{(0)}$. In Fig. 4 we plot $\delta_{T}^{(s)}$ (diamonds) and $\delta_{T}^{(c)}$ (circles) together with the prediction of the bulk theory Eq. (35) (full line) as a function of the packing fraction $\eta$.

Thus neither the bulk theory nor the numerical DFT results provide any hints for the occurrence of logarithmic terms in the expression of the surface tension of hard-sphere fluids in contact with curved hard surfaces.

For the contact density one knows a priori that for all values of $\eta$ both the DFT route and the bulk approach yield the same contact value $\rho\left(R_{i}^{+} \rightarrow \infty\right)=\beta p$ of the density profile of a hard-sphere fluid in the limit of the planar wall. For finite curvature we find that the contact densities obtained numerically from DFT (symbols) and analytically from our bulk theory for $\gamma\left(R_{s}^{(b)}\right)$ (full lines) are in very good quantitative agreement for all values of $\eta$, as shown in Fig. 5 for spherical walls and in Fig. 6 for cylindrical walls. Only for very large curvatures the actual DFT contact densities are slightly overestimated by the bulk theory.

For the spherical wall in Fig. 5 we also plot the contact density obtained from an empirical fit to simulation data (dotted lines) by Degreve et al. [6]. In terms of $R_{s}^{-1}$ this fit interpolates linearly between the planar wall limit within the Carnahan-Starling theory and the contact value of the pair correlation function $g(r)$ corresponding to $R / R_{s}=0.5$. At high packing fractions this fit deviates from our results in the planar wall limit due to the difference between the Carnahan-Starling equation of state and the Percus-Yevick compressibility pressure which underlies both our approaches. At very high packing fractions there are additional deviations because the simple linear fit does not capture the actual higher order terms in $R_{s}^{-1}$. Nonetheless, the overall agreement between our results and this fit is good.

\section{CONCLUSIONS AND SUMMARY}

Our analysis of the local structure and the thermodynamics of the hard-sphere fluid near hard curved substrates has lead to the following main results:

1. We have studied the curvature dependence of the surface tension of a hard-sphere fluid at hard spherical and cylindrical walls (Fig. 1) obtained by minimizing the Rosenfeld fundamental measure density functional. We have found no indications of logarithmic singularities in the expansion of the surface tension in terms of curvatures of spherical or cylindrical walls. The surface tension appears to be an analytic function of curvature.

2. Based on the Rosenfeld fundamental measure theory we have derived an analytical expression for the surface tension of a hard-sphere fluid close to hard, arbitrarily curved, convex walls in terms of their integrated mean and Gaussian curvatures, $H^{(b)}$ and $K^{(b)}$ (see Eq. (33)). This approach also does not render logarithmic singularities of the surface tension as a function of the radii of curvature. There is good agreement between the results of the density functional theory and the bulk theory which treats the curved wall as the surface of a particle of a second component in infinite dilution (Figs. 2 and 3). The Tolman length as a function of the packing fraction of the hard-sphere fluid is shown in Fig. 4.

3. Based on the sum rule for curved substrates we have obtained expressions for the contact values of the density profile of a hard-sphere fluid close to spherical (Eq. (36)) and cylindrical (Eq.(37)) walls. A comparison with the density functional results indicates, that the analytical expressions of the contact values are very reliable; only at rather high packing fractions they slightly overestimate the density functional values (Figs. 5 and 6 ) for large curvatures.

4. The ratio of the leading order terms in the expansion of the surface tension as a function of curvature, obtained from full minimization of the fundamental measure functional, for cylindrical and spherical symmetry equals 2 (see Table I). This is in agreement with the aforementioned bulk theory (Eq. (33)). This also agrees with the general feature of the Helfrich theory [28], in which for a sphere the contribution to the surface tension linear in the curvature is twice as large as for a cylinder.

In Ref. [41] the arguments in favor of and against the existence of logarithmic singularities in the curvature dependence of the surface tension of fluids have been reviewed. Our present results indicate that for a hard-sphere fluid near hard curved substrates such logarithmic singularities either do not exist or their influence is negligible.

It would be of interest to investigate the issue of analyticity of the surface tension via a diagrammatic expansion of the contact value of the density profile $[42,43]$. Within this approach our preliminary calculations show, that in the lowest order in density the diagrammatic expansion of the contact value of the density profile indeed leads to a surface 
tension analytical in curvature. However, the extension of this analysis to higher orders in densities is technically involved and is postponed to future work.

Thus our findings indicate that non-analyticities of the curvature dependence of surface tensions arise only via dispersion forces acting on the fluid particles [4] or via the onset of drying or wetting transitions on curved substrates [44]

\section{ACKNOWLEDGMENTS}

It is a pleasure to thank Bob Evans for stimulating discussions.

[1] A. Poniewierski and J. Stecki, J. Chem. Phys. 106, 3358 (1997).

[2] A. Samborski, J. Stecki, and A. Poniewierski, J. Chem. Phys. 98, 8958 (1993).

[3] J. Stecki and S. Toxvaerd, J. Chem. Phys. 93, 7342 (1990).

[4] T. Bieker and S. Dietrich, Physica A 252, 85 (1998).

[5] C. Bauer and S. Dietrich, Phys. Rev. E 62, 2428 (2000).

[6] L. Degreve and D. Henderson, J. Chem. Phys. 100, 1606 (1994).

[7] D. Henderson, S. Sokołowski, and A. Patrykiejew, Mol. Phys. 85, 745 (1995).

[8] M.P. Moody and P. Attard, J. Chem. Phys. 115, 8967 (2001).

[9] A.E. van Giessen, E.M. Blokhuis, and D.J. Bukman, J. Chem. Phys. 108, 1148 (1998).

[10] D.W. Oxtoby, J. Phys.: Condens. Matter 4, 7627 (1992).

[11] P.R. ten Wolde and D. Frenkel, J. Chem. Phys. 109, 9901 (1998).

[12] J. Barret, J. Chem. Phys. 111, 5938 (1999).

[13] R. Roth, B. Götzelmann, and S. Dietrich, Phys. Rev. Lett. 83, 448 (1999).

[14] R. Roth, R. Evans, and S. Dietrich, Phys. Rev. E 62, 5360 (2000).

[15] R. Roth, R. Evans, and A.A. Louis, Phys. Rev. E 64, 051202 (2001).

[16] D. Beysens, J.-M. Petit, T. Narayanan, A. Kumar, and M.L. Broide, Ber. Bunsenges. Phys. Chem. 98, 382 (1994), and references therein.

[17] A.J. Archer, R. Evans, and R. Roth, Europhys. Lett. 57, 526, (2002).

[18] F. Schlesener, A. Hanke, and S. Dietrich, J. Stat. Phys., 110, 981 (2003).

[19] A. Hanke and S. Dietrich, Phys. Rev. E 59, 5081 (1999).

[20] K. Lum, D. Chandler, and J.D. Weeks, J. Phys. Chem. 103, 4570 (1999).

[21] K. Katsov and J.D. Weeks, J. Phys. Chem. 105, 6738 (2001).

[22] P. Schofield and J.R. Henderson, Proc. Roy. Soc. Lond. A 379, 231 (1982).

[23] J.R. Henderson and P. Schofield, Proc. Roy. Soc. Lond. A 380, 211 (1982).

[24] J.R. Henderson, Mol. Phys. 50, 741 (1983).

[25] J.R. Henderson and J.S. Rowlinson, J. Phys. Chem. 88, 6484 (1984).

[26] J.R. Henderson, in Fluid Interfacial Phenomena, edited by C.A. Croxton (Wiley, Chichester, 1986 ), p. 555.

[27] R.C.J. Tolman, J. Chem. Phys. 17, 333 (1949).

[28] W. Helfrich, Z. Naturforsch. C 28, 693 (1973).

[29] F. David, in Statistical Mechanics of Membranes and Surfaces, edited by D. Nelson, T. Piran, and S. Weinberg (World Scientific, Singapore, 1989), p. 157.

[30] R. Lipowsky, in Handbook of Biological Physics, edited by R. Lipowsky and E. Sackmann, Vol. 1, (Elsevier, Amsterdam, 1995), p. 521.

[31] K. R. Mecke and S. Dietrich, Phys. Rev. E 59, 6766 (1999).

[32] C. Fradin, D. Luzet, D. Smilgies, A. Braslau, M. Alba, N. Boudet, K. Mecke, and J. Daillant, Nature 403, 871 (2000).

[33] Y. Rosenfeld, Phys. Rev. Lett. 63, 980 (1989).

[34] Y. Rosenfeld, J. Chem. Phys. 98, 8126 (1993).

[35] M. Dijkstra, R. van Roij, and R. Evans, Phys. Rev. E 59, 5744 (1999).

[36] J.R. Henderson, J. Chem. Phys. 116, 5039 (2002).

[37] Y. Rosenfeld, Phys. Rev. E 50, R3318 (1994).

[38] Y. Rosenfeld, Mol. Phys. 86, 637 (1995).

[39] H. Reiss, H.L. Frisch, E. Helfand, and J.L. Lebowitz, J. Chem. Phys. 32, 119 (1960).

[40] R. Roth and S. Dietrich, Phys. Rev. E 62, 6926 (2000). 
[41] J.S. Rowlinson, J. Phys.: Condens. Matter 6, A1 (1994).

[42] B.R.A. Nijboer and L. van Hove, Phys. Rev. 85, 777 (1952).

[43] J. Stecki and S. Sokołowski, Phys. Rev. A 18, 2361 (1978).

[44] R. Evans, R. Roth, and P. Bryk, unpublished (2003).

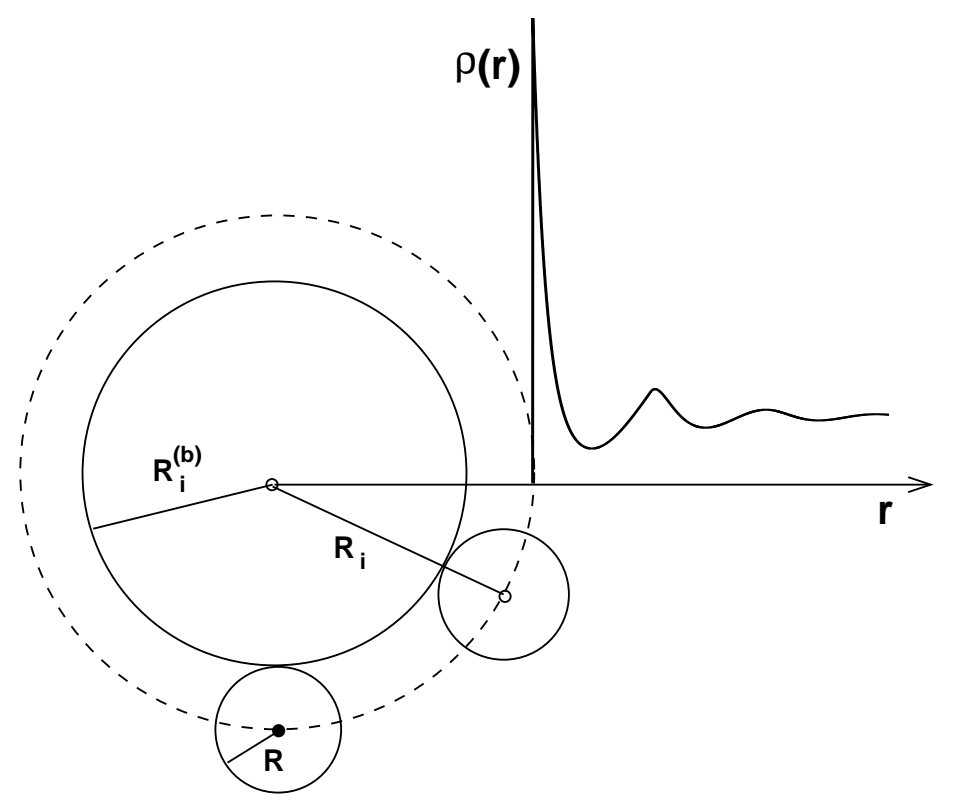

FIG. 1. Geometry of a hard-sphere fluid in contact with a hard spherical $(i=s)$ or cylindrical $(i=c)$ wall with radius $R_{i}^{(b)}=R_{i}-R$ and volume $V^{(b)}$. While the density profile $\rho(r)$ attains its contact value at $r=R_{i}$, we choose the wall, i.e., the surface with radius $R_{i}^{(b)}$ as dividing surface for the calculation of the surface tension. 

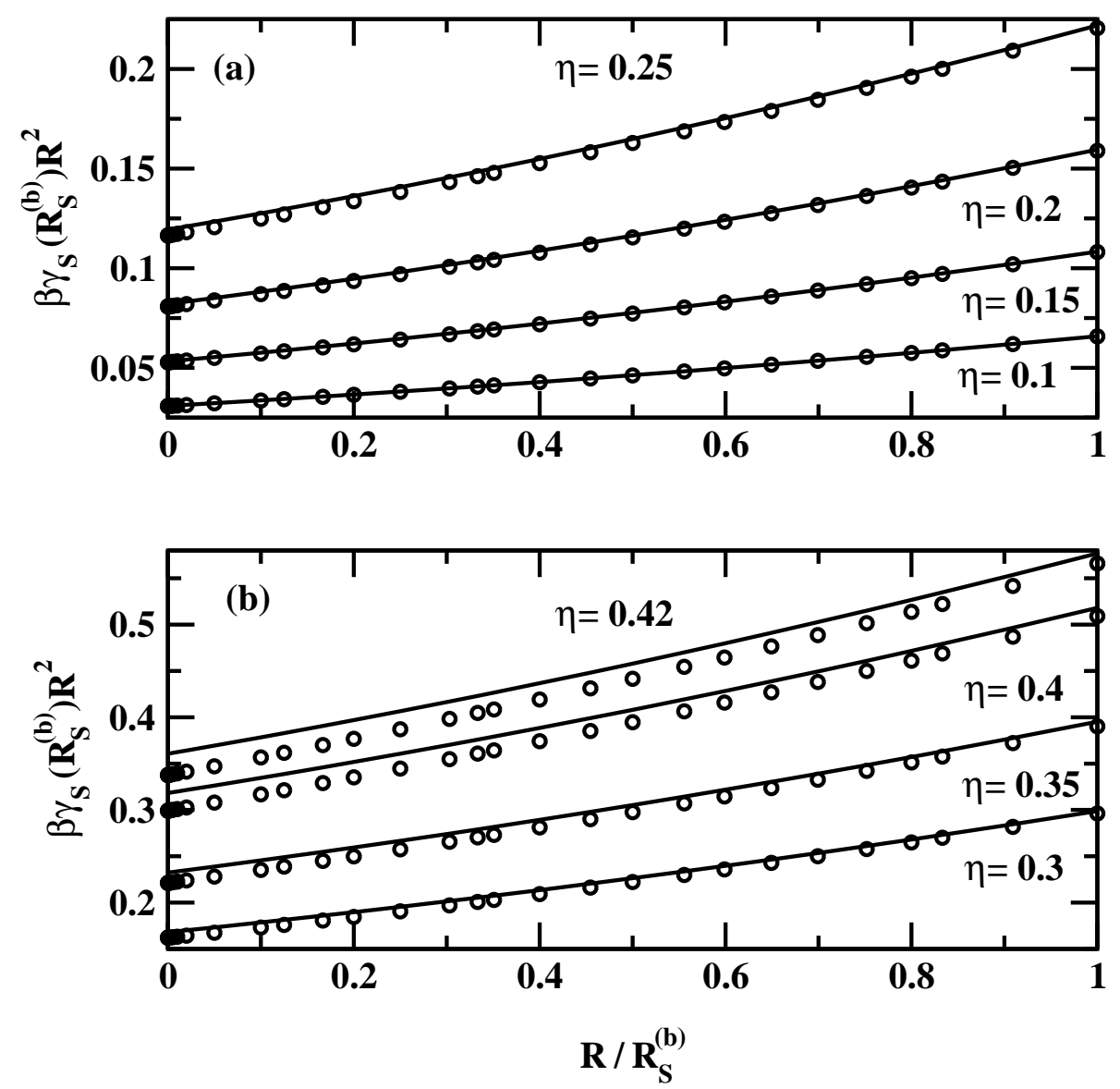

FIG. 2. The surface tension of a fluid of hard spheres with radius $R$ near a hard spherical wall of radius $R_{s}^{(b)}$. Symbols denote results obtained from direct minimization of the Rosenfeld functional, whereas the full lines are predictions of the bulk theory (see Eq. (33)). Small packing fractions $(\eta=0.1, \ldots, 0.25)$ are shown in (a) and large packing fractions $(\eta=0.3, \ldots, 0.42)$ in $(\mathrm{b})$. The agreement between both routes is excellent for low packing fractions. Towards high values of $\eta$ the level of quantitative agreement decreases, while the bulk theory still reproduces qualitatively the DFT results. 

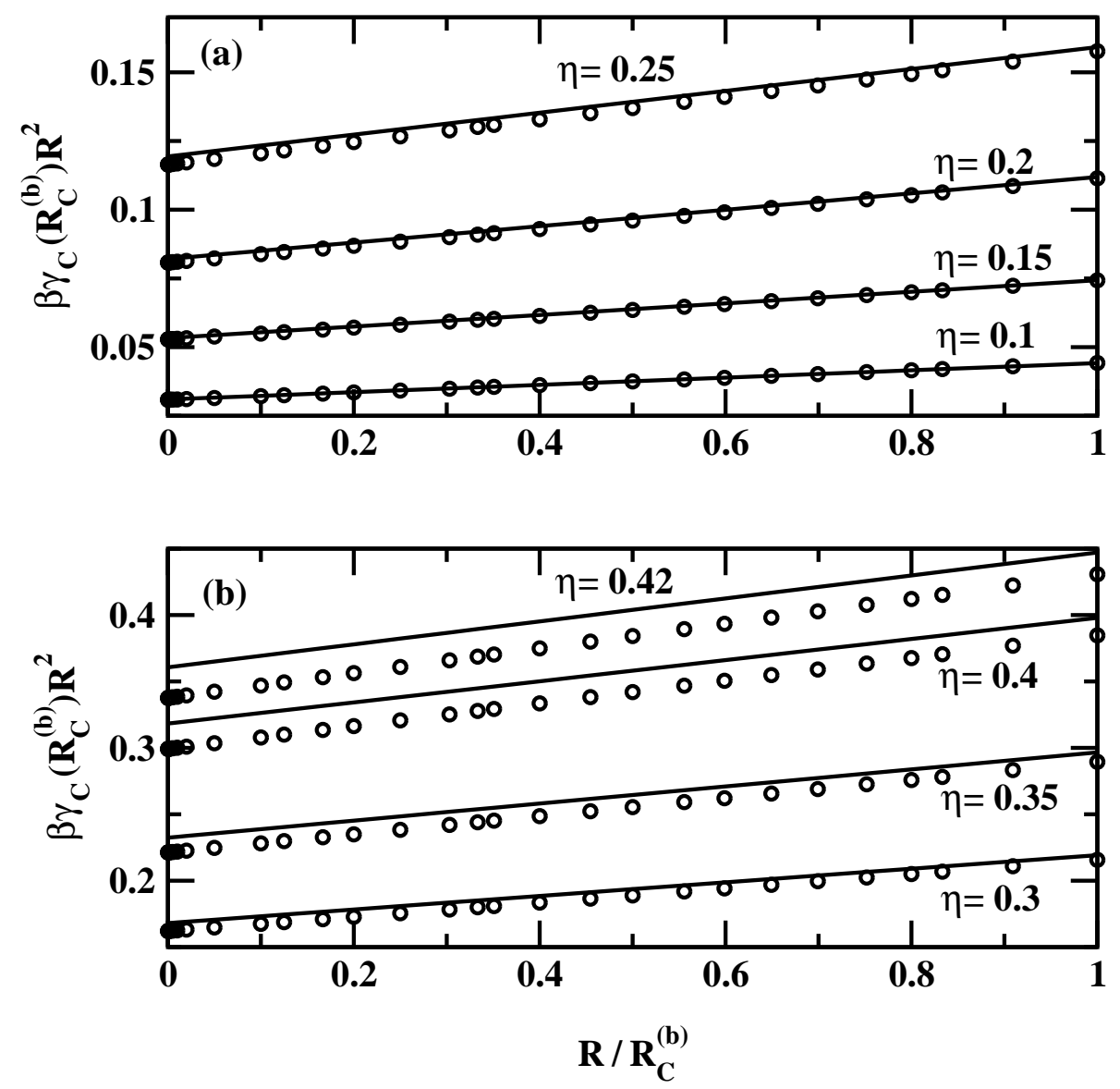

FIG. 3. The surface tension of a fluid of hard spheres with radius $R$ near a hard cylindrical wall of radius $R_{c}^{(b)}$. Symbols denote results obtained from direct minimization of the Rosenfeld functional, whereas the full lines are predictions of the bulk theory (see Eq. (33)). Small packing fractions $(\eta=0.1, \ldots, 0.25)$ are shown in (a) and large packing fractions $(\eta=0.3, \ldots, 0.42)$ in $(\mathrm{b})$. The agreement between both routes is excellent for low packing fractions. Towards high values of $\eta$ the level of quantitative agreement decreases, while the bulk theory still reproduces qualitatively the DFT results. 


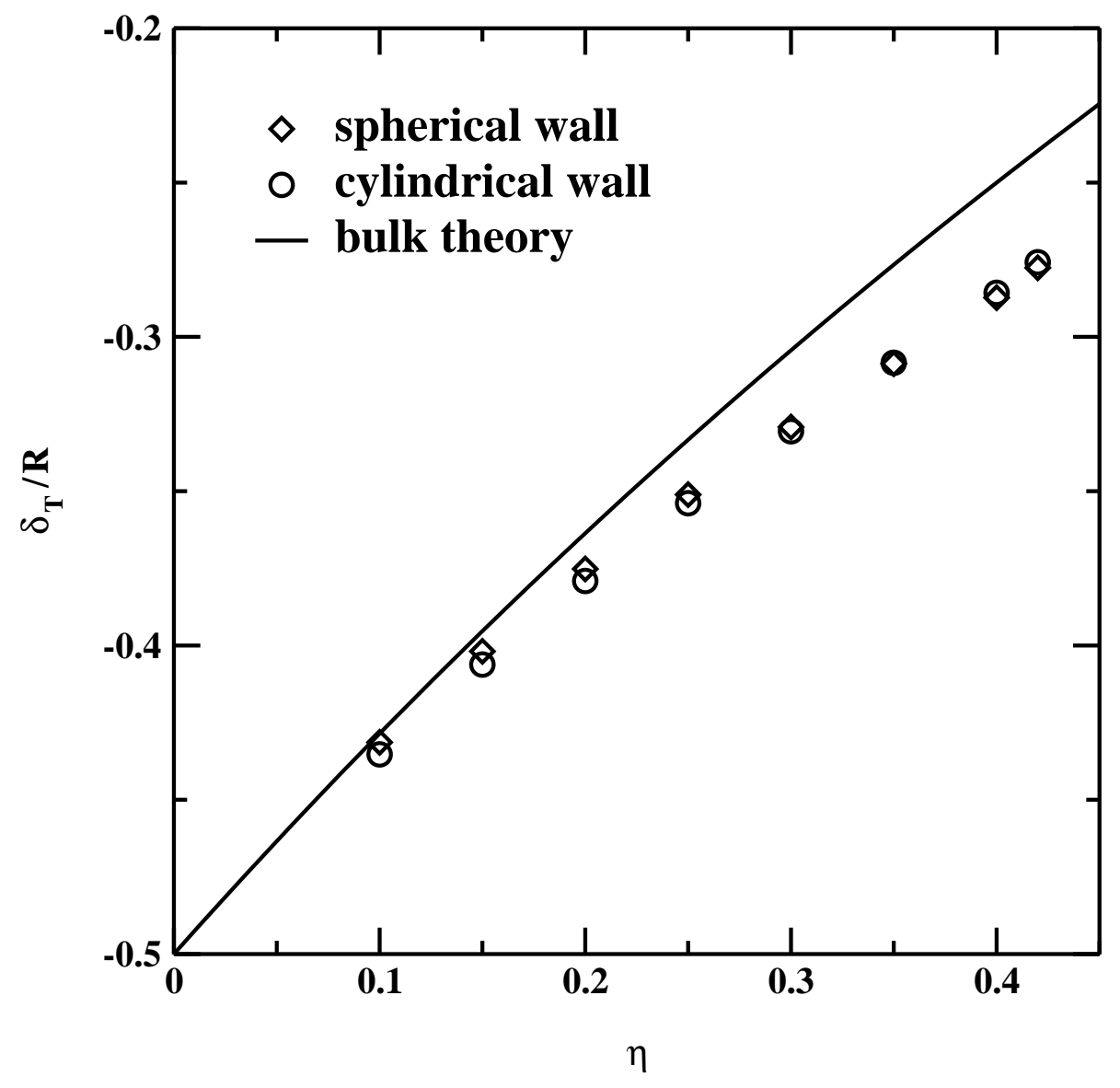

FIG. 4. The Tolman length $\delta_{T}$ of a fluid of hard spheres with radius $R$ at curved hard walls as a function of the packing fraction $\eta$. Diamonds and circles denote results for spherical and cylindrical walls, respectively, while the full line correspond to the prediction of the bulk theory (Eq. (35)). 

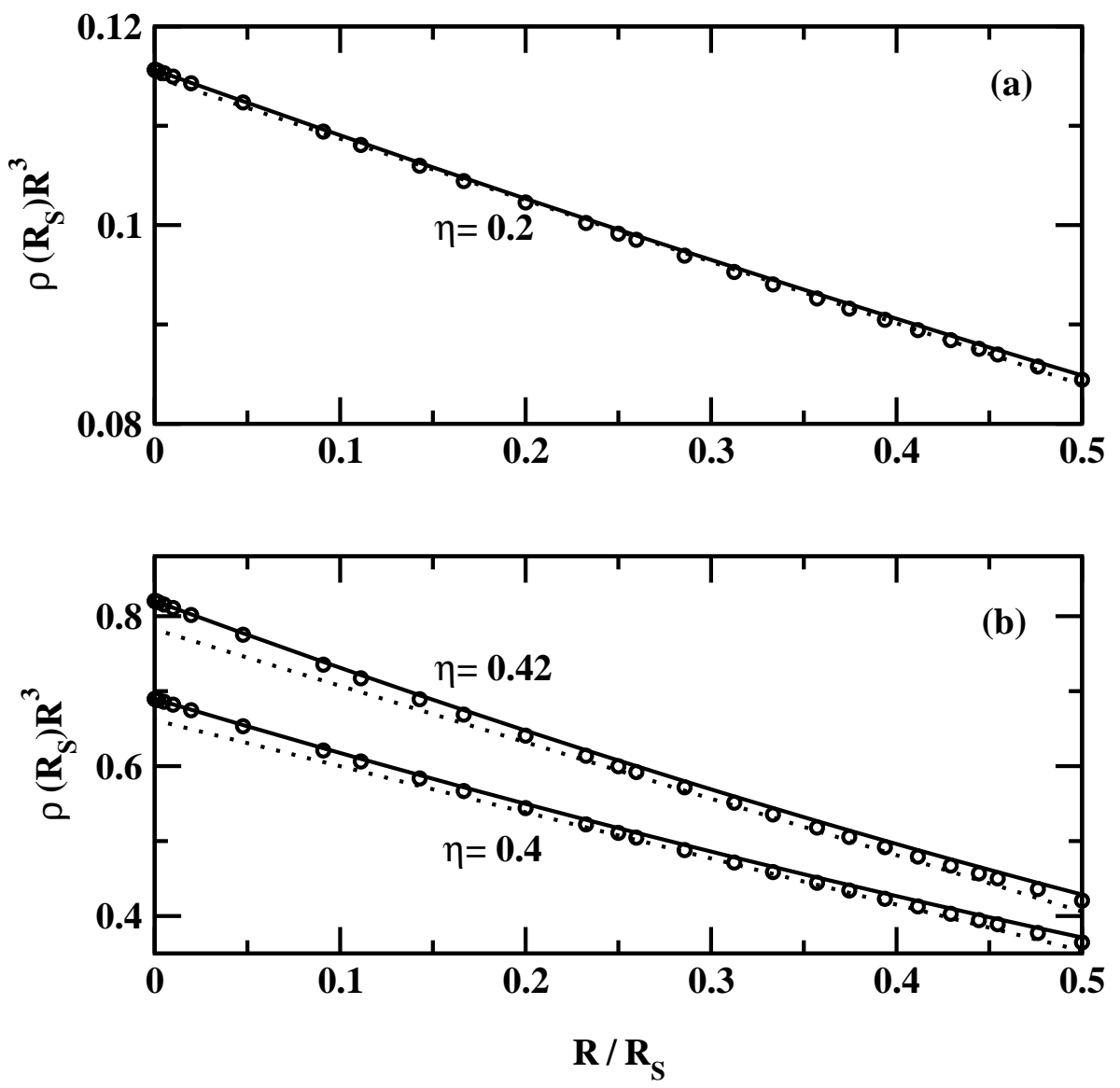

FIG. 5. The contact values of the density profile of a fluid of hard spheres with radius $R$ at a hard spherical wall with radius $R_{s}^{(b)}=R_{s}-R$ as obtained from direct minimization of the Rosenfeld functional (symbols), from the bulk theory (Eq. (36)) (solid lines), and from a semi-empirical parameterization [6] (dotted lines). The results for the packing fraction $\eta=0.2$ are given in (a) and for $\eta=0.4$ and $\eta=0.42$ in (b). Even for high packing fractions the agreement between the DFT results and the predictions of the bulk theory is very good. 

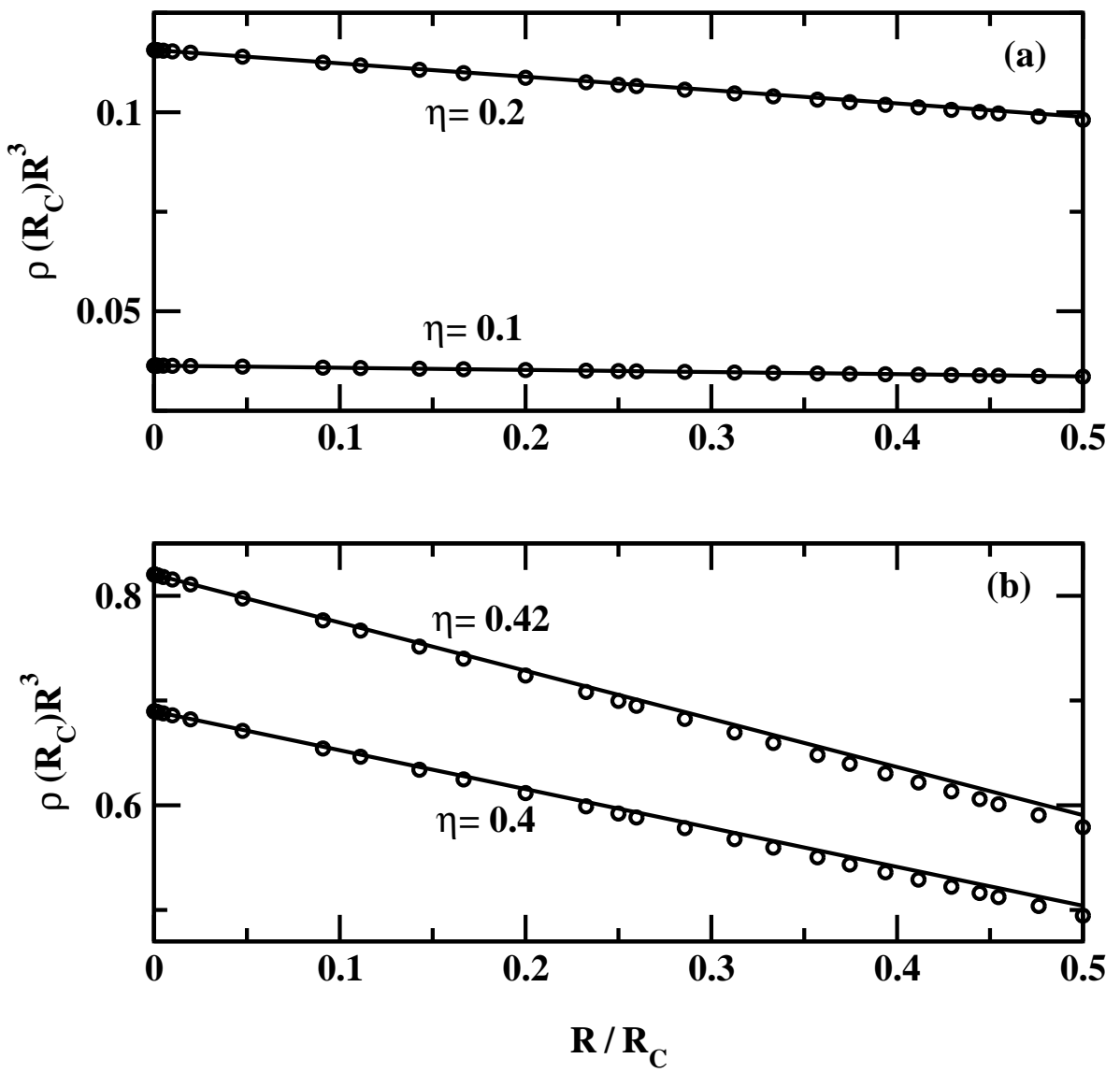

FIG. 6. The contact values of the density profile of a fluid of hard spheres with radius $R$ at a hard cylindrical wall with radius $R_{c}^{(b)}=R_{c}-R$ as obtained from direct minimization of the Rosenfeld functional (symbols) and from the bulk theory (Eq. (37)) (solid lines). The results for the packing fractions $\eta=0.1$ and $\eta=0.2$ are given in (a) and for $\eta=0.4$ and $\eta=0.42$ in (b). Even for high packing fractions the agreement between the DFT results and the predictions of the bulk theory is very good. 
TABLE I. Values of $a_{s}^{(0)}, a_{s}^{(1)}, a_{s}^{(2)}$ and $a_{c}^{(1)}$ as obtained from least square fit to DFT data of the surface tension of a hard-sphere fluid in contact with a spherical and cylindrical hard wall for various values of the packing fraction $\eta$. Note that Eq. (33) predicts $\frac{a_{s}^{(1)}}{a_{c}^{(1)}}=2$ for all $\eta$.

\begin{tabular}{|c|c|c|c|c|c|}
\hline$\eta$ & $a_{s}^{(0)} R^{2}=a_{c}^{(0)} R^{2}$ & $a_{s}^{(1)} R$ & $a_{s}^{(2)}$ & $a_{c}^{(1)} R$ & $a_{s}^{(1)} / a_{c}^{(1)}$ \\
\hline$\overline{0.10}$ & 0.03083 & 0.02660 & 0.00837 & 0.01342 & 1.98 \\
\hline 0.15 & 0.05281 & 0.04245 & 0.01286 & 0.02145 & 1.98 \\
\hline 0.20 & 0.08078 & 0.06061 & 0.01754 & 0.03062 & 1.98 \\
\hline 0.25 & 0.11643 & 0.08175 & 0.02235 & 0.04121 & 1.98 \\
\hline 0.30 & 0.16213 & 0.10674 & 0.02725 & 0.05360 & 1.99 \\
\hline 0.35 & 0.22127 & 0.13660 & 0.03229 & 0.06823 & 2.00 \\
\hline 0.40 & 0.29924 & 0.17195 & 0.03800 & 0.08548 & 2.01 \\
\hline 0.42 & 0.33761 & 0.18747 & 0.04084 & 0.09312 & 2.01 \\
\hline
\end{tabular}

